\title{
Las micro, pequeña y mediana empresa y sus adaptación a la normativa de seguridad y la salud en el trabajo
}

\author{
Micro, small and medium business and their adaptation to occupational \\ health and safety regulations
}

\author{
Fabiola Falconi Agapito ${ }^{1}$, Alfonso Romero Baylon ${ }^{2}$
}

Recibido: Enero 2020 - Aprobado: Junio 2020 - Publicado: Junio 2020

\section{RESUMEN}

En el año 2017 según datos estadísticos oficiales, las MIPYME formales (micro, pequeña y mediana empresa) se estimaron en 1,9 millones, siendo la gestión empresarial el punto donde radica la fuerza laboral generada en el Perú. Sin embargo, también estarían relacionadas con el fracaso organizacional enfrentando retos continuamente, uno de los mayores es cómo abordar el cumplimiento legal. Para enfrentar estos retos y no fracasar se debe tener en cuenta:

- $\quad$ La cultura empresarial responsable y con acceso financiero.

- El entorno organizacional interno y externo con reglas claras y simples de cumplir.

- $\quad$ La adaptación a las normativas legales

El objetivo de la presente investigación es integrar las principales normas de SST vigentes junto a la información estadística del Instituto Nacional de Estadística e Informática-INEI, para ello es necesario comprender los beneficios del Sistema de Gestión de SST. En la fase inicial del artículo, presentaremos parámetros priorizando tres aspectos como son: 1) Diagnósticos de SST; 2) Marco Legal; 3) Sistema de Gestión de SST con estructura y pautas de aplicación. A continuación para toda MIPYME, presentaremos el análisis estadístico y los resultados, con ejemplo práctico para verificar la funcionalidad del sistema, que finalmente explicaremos en las conclusiones y recomendaciones.

Palabras claves: Diagnóstico inicial legal; sistema de gestión de seguridad y salud en el trabajo; MIPYME.

\section{ABSTRACT}

In 2017, according to official statistical data, the formal MIPYME (micro, small and medium-sized companies, for its initials in spanish), were estimated at 1.9 million, with business management being the point where the workforce generated in Peru. However, they would also be related to organizational failure facing challenges continuously, one of the biggest is how to approach legal compliance. To face these challenges and not fail, the following must be taken into account:

- Responsible business culture with financial access.

- $\quad$ Internal and external organizational environment with clear and simple compliance rules.

- $\quad$ Adaptation to legal regulations

The objective of this research is to try to integrate the main standards of OSH together with the statistical information National Institute of Statistics and Informatics-INEI, for this it is necessary to understand the benefits of the OSH Management System. In the initial phase of the article, we will present parameters prioritizing three aspects such as: 1) OSH diagnoses; 2) legal framework; 3) OSH Management System with structure and application guidelines. Below for all MIPYME, we will present the statistical analysis of the results with a practical example to verify the functionality of the system, which we will finally explain in the conclusions and recommendations.

Keywords: Initial legal diagnosis; occupational health and safety management system.

\footnotetext{
${ }^{1}$ Egresado de la Maestría en Gestión Integrada en Seguridad, Salud ocupacional y medio Ambiente, Facultad de Ingeniería Geológica, Minera, Metalúrgica y Geográfica, Universidad Nacional Mayor de San Marcos. Lima, Perú. E-mail: fabiolafalconil@hotmail.es

${ }^{2}$ Docente de la Facultad de Ingeniería Geológica, Minera, Metalúrgica y Geográfica, Universidad Nacional Mayor de San Marcos. Lima, Perú.

E-mail: aromerob@unmsm.edu.pe
} 


\section{INTRODUCCIÓN}

La implementación de un Sistema de Gestión para las empresas MIPYME en el Perú es fundamental. Primeramente, por la evidencia de los beneficios que se obtienen en diversos rubros, y la segunda, es la obligación por formalizarse, que en muchos casos es la razón principal de su implementación. Por ello, esta obligatoriedad normada por el Estado para el cumplimiento de las normas de SST, no da un espacio para un mayor análisis al momento de iniciar la implementación del sistema de gestión.

En esta investigación trataremos de establecer las modalidades para su implementación, identificando primero las causantes de la falta de un sistema de gestión de SST (Instituto de Seguridad Minera-ISEM, s. f.) en las MIPYMES, como son:

- No contar con personal que desarrolle la ejecución del sistema de seguridad.

- Limitación en el acceso a medios para la implementación del sistema de gestión.

- Acceso a información y oportunidades para una alineación oportuna en temas de SST.

En muchos casos los empleadores de las PYMES al desconocer los beneficios de la implementación de un sistema de SST como son la reducción de los accidentes (Fernandez, s. f.) y enfermedades, no toman la decisión de incluirlos en su productividad y rentabilidad.

A partir de la identificación, se podría explicar los comportamientos individuales de cada empresa para establecer la mejor manera de implementar el sistema de gestión de SST.

En ese sentido, para la detección de esta causante y posterior implementación del sistema de gestión, se requiere información estadística de accidentabilidad, datos de una empresa como modelo a implementar y resultados posteriores a la implementación.

En síntesis, conocer la situación actual mediante el diagnóstico inicial acorde a la normativa vigente, luego la determinación de los 9 (nueve) puntos de implementación particular, así como la deducción adecuada para cada MIPYME.

Por ello, es primordial que la implementación de un sistema de gestión de SST pueda estar al alcance con este modelo de la propia normativa vigente, de integración de las normativas actuales dadas por el Estado, permitirá que más MYPIMES puedan tener un resultado expectante. De modo que, la aplicación del modelo a describir permitirá la determinación de un sistema de gestión fuerte y con reducción en las pérdidas.

A lo mencionado en el párrafo anterior, se plantea como objetivo desarrollar un modelo de fácil seguimiento y listo para su uso en la implementación del sistema de gestión de SST para las MIPYME.

\section{METODOS}

El proceso de investigación comprende 4 fases de implementación y diagnóstico previo:

- Revisión y recopilación de información

- Diagnostico situacional de línea base

- Planificación del Sistema de Gestión SST

- Implementación del Sistema de Gestión SST

Para que este modelo conceptual y teórico de gestión de SST funcione, cada MIPYME debe de tener un diagnóstico y reunión de implementación previa. Para ello, se eligió una empresa PYME dedicada a la exploración que forma parte de la actividad minera en Lima-Provincia.

La información que se utilizó en esta investigación fue proporcionada por la propia empresa y se contó con los datos estadísticos oficiales desde el Ministerio de Energía y Minas (Ministerio de Energía y Minas - ÍNDICES DE FRECUENCIA Y SEVERIDAD SOBRE ACCIDENTES DE TRABAJO - Minería, s. f.), iniciando con la siguiente Tabla 1.

Tabla 1. Población

\begin{tabular}{ccc}
\hline $\begin{array}{c}\text { Trabajadores según } \\
\text { grupo ocupacional }\end{array}$ & $\begin{array}{c}\text { Total de } \\
\text { trabajadores }\end{array}$ & Muestra \\
\hline Gerente & 1 & 1 \\
Jefes de Área & 4 & 4 \\
Supervisores & 08 & 08 \\
Perforistas & 10 & 10 \\
Ayudantes & 35 & 35 \\
Otros & 03 & 03 \\
Total & $\mathbf{6 1}$ & $\mathbf{6 1}$
\end{tabular}

Fuente. Fabiola Falconi, 2018

\subsection{Metodología de revisión y recopilación de información}

La búsqueda se inicia con la recopilación de la documentación con base en la normativa peruana actual, así como en experiencias propias respecto a las dificultades que tienen las MIPYMES para la implementación de la SST, lo que permitió formular las hipótesis correspondientes.

Con respecto a las fuentes de esta investigación, inicie con la revisión de las normas legales vigentes; posteriormente, focalice la búsqueda en investigaciones especializadas e institucionales, que tengan un patrón y origen similar, contando con la asesoría de diferentes profesionales y profesores académicos (tesis, trabajos especiales, entre otros).

A continuación, se realizó la selección minuciosa del material bibliográfico, seleccionado con relación al área de estudio (ver Tabla 2). 
Tabla 2. Metodología de Investigación

\begin{tabular}{|c|c|}
\hline \multicolumn{2}{|c|}{ Pasos de la descripción } \\
\hline Planear & $\begin{array}{l}\text { Planificar todas las actividades que van a formar parte del Sistema } \\
\text { de Gestión de seguridad y salud, identificar los requerimientos, } \\
\text { procedimientos, responsables, funciones y procesos a los cuales } \\
\text { voy a aplicar en la empresa. }\end{array}$ \\
\hline Hacer & Implementar los procedimientos previamente establecidos. \\
\hline Verificar & $\begin{array}{l}\text { Comprobar que se ha hecho como se ha planificado y diseñar } \\
\text { auditorias tanto internas como externas, para verificar que los } \\
\text { procedimientos establecidos se estén cumpliendo. }\end{array}$ \\
\hline Actuar & $\begin{array}{l}\text { Tomar acciones de mejora en función de las revisiones periódicas, } \\
\text { que se hagan ya sea interno o externo. }\end{array}$ \\
\hline
\end{tabular}

Fuente. (Instituto de Seguridad Minera-ISEM, s. f.)

\subsection{Diagnostico situacional de línea base}

Para establecer un punto de partida en el sistema de gestión de seguridad que requiere cada empresa, se ejecutará a través de un diagnóstico inicial, el cual es un punto de partida que nos dará el grado de cumplimiento en cuanto al sistema de gestión de SST.

Este resultado se analiza y expone a la dirección y gerencia de la empresa para tener el punto de partida y tener como referencia durante el proceso de implantación de la presente propuesta.

A continuación mostraré la guía que se seguirá para verificación y revisar el grado de cumplimiento de las normas, vigentes en materia de SST, que nos guiará a los siguientes lineamientos:

- Cada ítem contara con un puntaje establecido de acuerdo a los criterios.

- Se calificara acorde a cada rubro donde se obtendrá la situación de cada empresa en base a su rubro.

- La documentación deberá ceñirse a los ya establecidos en cada norma, los que sustentaran el sistema.

- Presentación de resultados, evidencia de actividades ejecutadas, registros, programas de cumplimiento, entre otros.

\subsection{Planificación del sistema de gestión de SST}

Se realizó una evaluación inicial situacional de la empresa de la empresa minera con respecto a trabajos de exploración minera.

En base a estos diagnósticos se estableció los lineamientos que se requiere para iniciar la implementación, ello se realizó acompañado de un cronograma y plan de trabajo que fue revisado por la gerencia, manteniendo la búsqueda de su cumplimiento acorde a lo estipulado por el estado.

Se realizó los diagnósticos basados en las normas:

- $\quad$ Ley N 29783

- D.S 0052012 TR

- $\quad \mathrm{RM} \mathrm{N}^{\circ} 050-2013-\mathrm{TR}$,
Los resultados se analizaron y fueron expuestos a la dirección y gerencia de la empresa a continuación se muestra los diagnósticos resumen basados en las normas (Ver apéndice 1, apéndice 2).

\subsection{Implementación del sistema de gestión de SST}

Con la finalidad de establecer lineamientos necesarios en el proceso de adecuación a la gestión que mantendrá una mejora necesaria en seguridad y salud en el trabajo, para ello el liderazgo permanente y compromiso de toda la línea de mando a todos los niveles de la empresa, así mismo; en conjunto con la dirección quien proporciono las medidas que se requirieron para asegurar que todo miembro de la organización participe de la implementación y se cree el compromiso requerido en base a lo siguiente:

- Se elaboró el manual de organización y funciones (MOF), para cada puesto de trabajo en la organización y esta alineada a la implementación del Sistema.

- Se aseguró que cada miembro cuente con la formación en temas de Seguridad y la competencia para realizar sus labores.

- Contaron con los medios y recursos necesarios para realizar sus tareas en forma segura y saludable.

La implementación para el Sistema de Gestión de SST constó de 09 elementos, la misma que pasare a detallar en los siguientes puntos:

\section{Elemento 01 - Liderazgo y Compromiso Visible}

Elemento 02 - Concientización y Capacitación

Elemento 03 - Gestión de Riesgos y Manejo del Cambio

Elemento 04 - Controles Operacionales y Gestión de Acción Correctiva

Elemento 05 - Higiene y Salud Ocupacional

Elemento 06 - Requisitos Legales y Otros

Elemento 07 - Gestión de Incidentes

Elemento 08 - Respuesta para Emergencias y Manejo de Crisis

Elemento 09 - Evaluación del Desempeño y Gestión (Objetivos y Metas)

Nota: todos en base a los resultados del diagnóstico.

\section{RESULTADOS Y DISCUSIÓN}

Al revisar los resultados posteriores a la implementación de la presente investigación se demostró su efectividad de esta implementación para una gestión de SST para la PYME objeto del estudio.

Esta aplicación metodológica fue validada de manera parcial por (Zamora, s. f.). En su trabajo ante la comunidad 
andina: auditorías de verificación y (echevarría $\&$ adrián, 2016). Análisis de riegos en exploraciones mineras para implementar esta verificación del sistema en seguridad y salud ocupacional. Por ello, la metodología de implementación funciona obteniendo buenos resultados, como se mostrará en las conclusiones de la presente investigación.

Se aplicó el análisis estadístico en cada una de las normas, por estar identificados como más críticos y de obligatoriedad legal para el rubro de minería

Las valoraciones que se asignan en esta investigación, es dada en porcentajes para facilidad de lectura de resultados.

Siendo el objetivo principal la implementación del sistema, para alinear a la normativa actual y minimizar la ocurrencia de accidentes, enfermedades ocupacionales lo mostrado en el acápite 3.6, sustentara que la implementación de estos nueve elementos, son viables.

De esta implementación se pudo lograr que la Empresa cumpla con las normativas legales indicadas y de esta manera llegar a la reducción de accidentes en la empresa minera los cuales fueron favorables y aportaran a cada sector de manera positiva.

Teniendo en cuenta todos los pasos ejecutados para llegar a la implementación general, es necesario:

- Los resultados de la implantación y gestión de un sistema de gestión en seguridad y salud ocupacional en el trabajo, seguirán mejorando a través del tiempo.

- Estos resultados podrán permitir a esta y otras MYPIMES obtener una mayor eficiencia, eficacia y rentabilidad sus sistemas de gestión de SST, reduciendo sus accidentes y cumpliendo la norma.

Cada uno de los 09 elementos de implementación indicados en el ítem 2.5, debe ser acompañado por un riguroso seguimiento, de ello depende el cumplimiento de los objetivos trazados en cada uno de ellos y sobretodo generadas acciones a las desviaciones que se presenten.

Los resultados comparativos luego de la implementación se muestra en la figura 1 y figura 2.

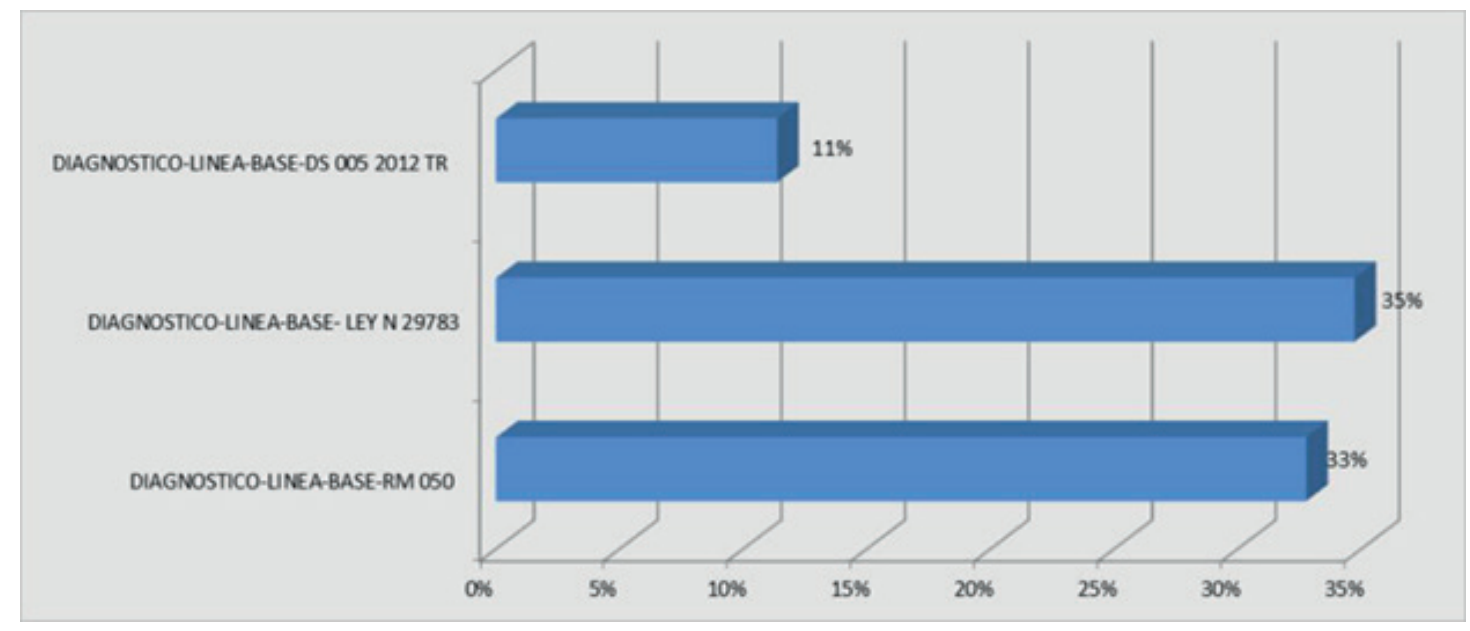

Figura 1. Cuadro resumen diagnósticos antes de la implementación de la gestión de SST Fuente. Fabiola Falconi, 2018

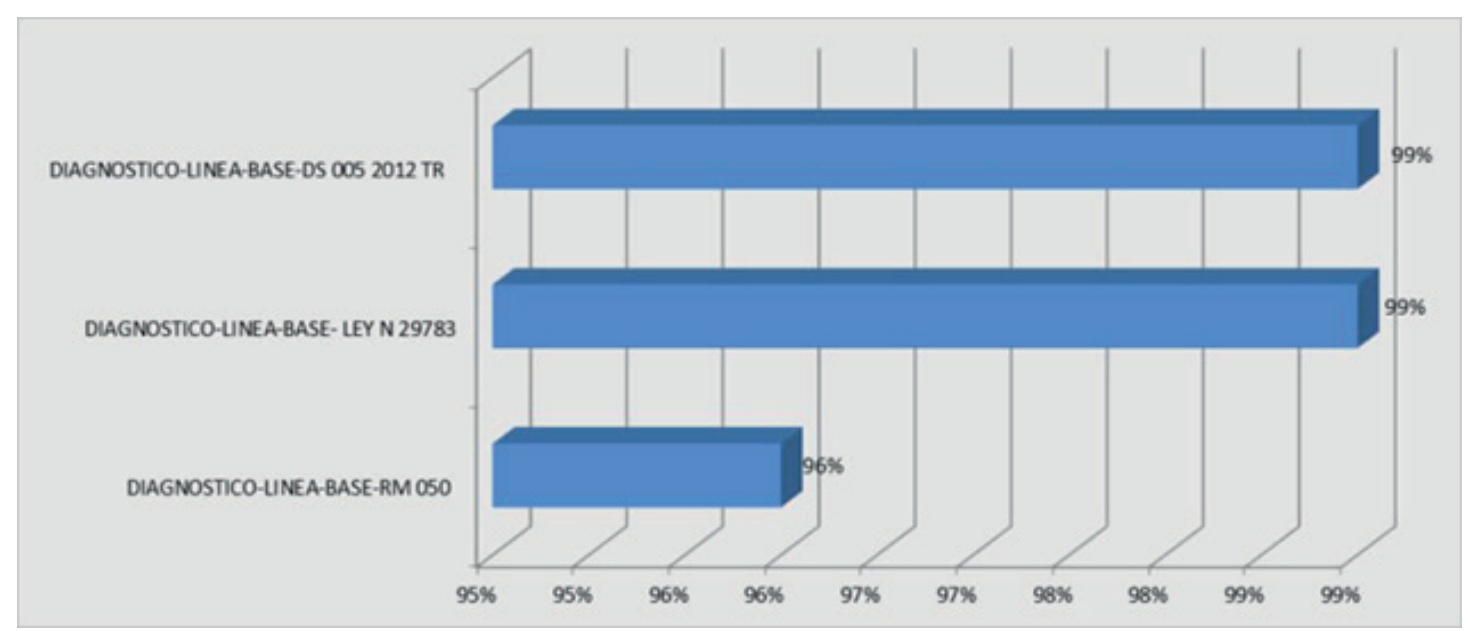

Figura 2. Cuadro resumen diagnóstico posterior a la implementación de la gestión de SST Fuente. Fabiola Falconi, 2019 
Todos los años cada empresa deberá considerar la necesidad de realizar nuevas evaluaciones para identificar las mejoras que requiera el sistema de gestión de SST, donde se deberá trazar los lineamientos necesarios para su cumplimiento. Toda revisión debe ser documentada, donde se especificara todas las necesidades identificadas, para cuando se implementen, se demuestre la mejora ejecutada al sistema y definir su permanencia. La organización establecerá la frecuencia en que se realizara estas evaluaciones, concertando como va mejorando el sistema, y comparándolas con las políticas, objetivos trazados y cumplidos, de esta manera identificaremos también las oportunidades de mejora.

\section{CONCLUSIONES}

- Existe una integración entre la parte teórica y práctica de gestión de SST que permita la implementación base para las MIPYME en el Perú, por lo que concluyo que el modelo funciona y puede aplicarse a toda empresa MIPYME.

- En el diagnóstico inicial del sistema de gestión de SST, se tiene cumplimientos de cumplimiento de $11 \%, 29 \%, 33 \%$ y $35 \%$ mientras que el mayor incumplimiento se presenta en los principios de planeamiento, aplicación operativa, control de información y documentos del sistema de gestión de seguridad.

- Según el último diagnóstico realizado en la empresa, se tiene casi un $96 \%$ y $99 \%$ del cumplimiento legal vigente, reflejando un gran cambio no solo en las estadísticas, sino también en la organización, demostrando la efectividad de la implementación con los 09 elementos, iniciando con el diagnóstico, que nos llevó a trazar metas y evitando perdidas de índole personal.

- El compromiso brindado por la alta dirección fue y debe continuar como uno de los factores para generar una buena gestión, en esta investigación aportaron a que los objetivos se cumplieran ya que ello permitió un efecto cascada en las Jefaturas, supervisión y trabajadores en general.

- La elaboración e implementación de cada uno de los 09 elementos desarrollados en un sistema de gestión de seguridad y salud ocupacional en el trabajo, llevara a la empresa no solo a cumplir con la normativa, si no también desarrolla en la empresa trabajadores líderes y experimentados, si no empresas con altos estándares y competitivas en el mercado, por ello se recomienda que haya un seguimiento por parte de todos los niveles de la organización y colocar responsables para verificar el cumplimiento de las mismas a fin de mantenerlos en el tiempo.

- Se propone que las MIPYME, comprueben con la aplicación del presente estudio su efectividad en la gestión de SST, utilizando como principal herramienta la fortaleza económica o ser incluido como futuros diagnósticos.
- Para la implementación del sistema de SST tener en cuenta la inversión que conlleve los siguientes puntos: EPP, capacitaciones, entrenamiento, materiales diversos, costos por salud ocupacional, de monitoreo de higiene y auditorías.

- Esta investigación puede ser aplicada a cualquier empresa que requiera implementar un sistema de gestión de seguridad y salud ocupacional en el trabajo, ya que según el rubro se puede seleccionar uno de los diagnósticos descrito en la investigación y aplicarlo a esta empresa.

\section{AGRADECIMIENTOS}

Mi agradecimiento a la Universidad Nacional Mayor de San Marcos, donde me forme y aprendí a buscar nuevos retos por alcanzar para el progreso de muchos, que en esta ocasión me permite desarrollar este artículo de investigación. Mi gratitud a los profesores y amigos en cada casa de estudio en donde buscamos aportar al crecimiento de la ciencia y la investigación.

\section{REFERENCIAS}

Decreto Supremo $N^{\circ}$ 005-2012-TR - Norma Legal Diario Oficial El Peruano. (s. f.). Recuperado 18 de junio de 2020, de https://busquedas.elperuano.pe/normaslegales/reglamentode-la-ley-n-29783-ley-de-seguridad-y-salud-en-e-decretosupremo-n-005-2012-tr-781249-1/

Echevarría, O., \&Adrián, J. (2016). Análisis de riesgos en exploraciones mineras para implementar un sistema de seguridad y salud ocupacional en el Perú. Universidad San Ignacio de Loyola. http://repositorio.usil.edu.pe/handle/USIL/2427

Fernandez, J. P. (s. f.). SGS Perú: Mayoría de accidentes en minería son causa de una frágil cultura de prevención. Energiminas. Recuperado 18 de junio de 2020, de https:// energiminas.com/sgs-peru-mayoria-de-accidentes-enmineria-son-causa-de-una-fragil-cultura-de-prevencion/

Gestión, R. (2020, febrero 14). ¿Qué requisitos deben cumplir las empresas para evitar sanciones por Seguridad y Salud en el Trabajo? Gestión. https://gestion.pe/economia/conque-requisitos-deben-cumplir-las-empresas-para-evitarsanciones-por-seguridad-y-salud-en-el-trabajo-noticia/

Instituto de Seguridad Minera-ISEM. (s. f.). Recuperado 18 de junio de 2020, de https://www.isem.org.pe/portal/files/ recurso/revista/87.pdf

LeydeSeguridadSaludTrabajo(s.f.). Recuperado 18dejuniode2020, de https://www.sunafil.gob.pe/images/docs/normatividad/ LEYDESEGURIDADSALUDTRABAJO-29783.pdf

dMinisterio de Energía y Minas-Índices de frecuencia y severidad sobre accidentes de trabajo - Minería. (s. f.). Recuperado 18 de junio de 2020, de http://www.minem.gob. pe/_estadistica.php?idSector $=1 \&$ idEstadistica $=12486$

Zamora, L. V. (s. f.). Sistemas de gestion de seguridad y salud en el trabajo en la comunidad andina: auditorías de verificación. http://rabida.uhu.es/dspace/bitstream/ handle/10272/12607/Sistemas_de_gestion_de_seguridad. pdf;jsessionid=3CA550FA4B33BD2F3D151D44B17CB27 4 ? sequence $=2$ 


\section{APÉNDICE}

Apéndice 1. Diagnostico ley 29783 - gestión de SST

\begin{tabular}{|c|c|c|c|c|c|}
\hline & \multirow{4}{*}{\multicolumn{3}{|c|}{$\begin{array}{l}\text { DIAGNOSTICO DE LINEA BASE LEY N²9783 Y SU MODIFICATORIA LA } \\
\text { LEY N } 30222\end{array}$}} & \multicolumn{2}{|c|}{ Elaborado por: F. Falconi } \\
\hline & & & & \multicolumn{2}{|c|}{ Revisado por: } \\
\hline & & & & \multicolumn{2}{|c|}{ Código: SSOMA } \\
\hline & & & & \multicolumn{2}{|c|}{ Fecha: JUNIO 2017} \\
\hline \multicolumn{6}{|c|}{ Razón Social Empresa: } \\
\hline \multicolumn{6}{|c|}{ RUC: } \\
\hline \multicolumn{6}{|c|}{ Domicilio Fiscal: } \\
\hline \multicolumn{6}{|c|}{ ESPECIFICAR EL TIPO DE ACTIVIDAD : } \\
\hline \multirow{3}{*}{$\begin{array}{l}\text { Requisitos } \\
\text { Obligatorios }\end{array}$} & \multirow{2}{*}{$\begin{array}{l}\text { Plataforma para la evaluación de un sistema para la prevención } \\
\text { de pérdidas y cumpliendo la normativa vigente. }\end{array}$} & \multicolumn{2}{|c|}{$\begin{array}{l}\text { ¿Cumple? } \\
\text { (marcar "X") }\end{array}$} & \multicolumn{2}{|c|}{ Observaciones } \\
\hline & & $\mathrm{Si}$ & No & N/A & \\
\hline & 1. POLITICA & & & & \\
\hline 1 & 1.1 Cuenta la organización con una política de prevención? & & & & \\
\hline 2 & $\begin{array}{l}\text { 1.2 Expresa el compromiso en cuanto a: Prevención de daños a la salud, } \\
\text { Cumplimiento legal, Consulta y Participación de los trabajadores y Mejora } \\
\text { Continua? }\end{array}$ & & & & \\
\hline \multirow[t]{2}{*}{3} & 1.3 Se encuentra exhibida y difundida entre el personal? & & & & \\
\hline & 2. ALCANCE DEL SISTEMA & & & & \\
\hline \multirow[t]{3}{*}{4} & $\begin{array}{l}\text { 2.1 El alcance del SGSST abarca TODA actividad que desarrolla los trabajadores } \\
\text { dentro o fuera de las instalaciones, dentro o fuera del horario de trabajo bajo } \\
\text { autoridad del empleador? }\end{array}$ & & & & \\
\hline & 3. PLANIFICACIÓN & & & & \\
\hline & 3.1 Estudio línea base & & & & \\
\hline \multirow[t]{2}{*}{5} & 3.1.1 Se ha realizado un estudio de línea base del Sistema Gestión de la SST? & & & & \\
\hline & 3.2 Identificación de Peligros y Evaluación de Riesgos (IPER) & & & & \\
\hline 6 & 3.2.1 Cuenta con un Procedimiento y Metodología para desarrollar los IPER? & & & & \\
\hline 7 & $\begin{array}{l}\text { 3.2.2 Tiene desarrollado los IPER para todas sus AREAS y PUESTOS TRABAJO } \\
\text { de la organización? }\end{array}$ & & & & \\
\hline 8 & $\begin{array}{l}\text { 3.2.3 Los registros IPER guardan relación con las actividades de riesgo que ha } \\
\text { indicado y que desarrolla en las instalaciones. }\end{array}$ & & & & \\
\hline 9 & $\begin{array}{l}\text { 3.2.4 Se actualiza el IPER por lo menos una vez al año y cuando ocurren } \\
\text { accidentes o incidentes de alto potencial y cuando ocurran cambios en las } \\
\text { condiciones de trabajo? }\end{array}$ & & & & \\
\hline 10 & 3.2.5 Tiene publicado los MAPAS DE RIESGO en cada área de la empresa? & & & & \\
\hline \multirow[t]{2}{*}{11} & $\begin{array}{l}\text { 3.2.6 Se vienen tratando los riesgos con la implementación de las medidas de } \\
\text { control sugeridas en el IPER? }\end{array}$ & & & & \\
\hline & 3.3 Objetivo, Metas y Programas & & & & \\
\hline 12 & 3.3.1 Se han definido Objetivos y Metas en el Sistema Gestión de SST? & & & & \\
\hline 13 & $\begin{array}{l}\text { 3.3.2 Los Objetivos y Metas en el Sistema Gestión de SST se encuentran } \\
\text { publicados en lugar visible? }\end{array}$ & & & & \\
\hline \multirow[t]{3}{*}{14} & 3.3.3 Cuenta con un Programa Anual de la SST? & & & & \\
\hline & 4. IMPLEMENTACIÓN Y OPERACIÓN & & & & \\
\hline & 4.1 Recursos, Funciones, Responsabilidad y Autoridad & & & & \\
\hline 15 & 4.1.1 Cuenta con un Organigrama actualizado? & & & & \\
\hline 16 & $\begin{array}{l}\text { 4.1.2 Cuenta con un MANUAL DE FUNCIONES que incluya todos los puestos de } \\
\text { trabajo? }\end{array}$ & & & & \\
\hline 17 & $\begin{array}{l}\text { 4.1.3 Se considera en las funciones de los Puestos Trabajo de nivel de liderazgo y } \\
\text { trabajadores en general su responsabilidad en materia de SST? }\end{array}$ & & & & \\
\hline
\end{tabular}




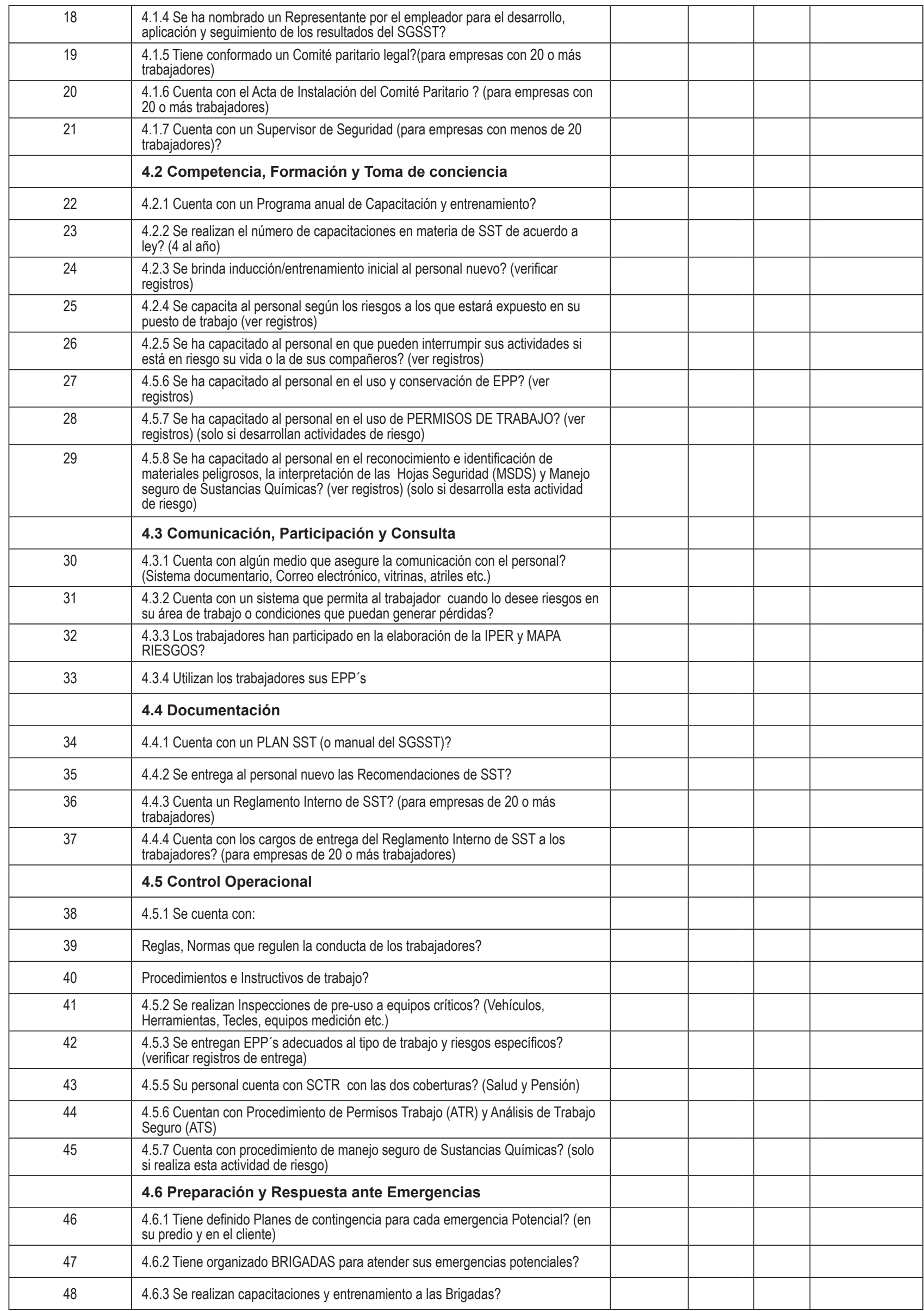




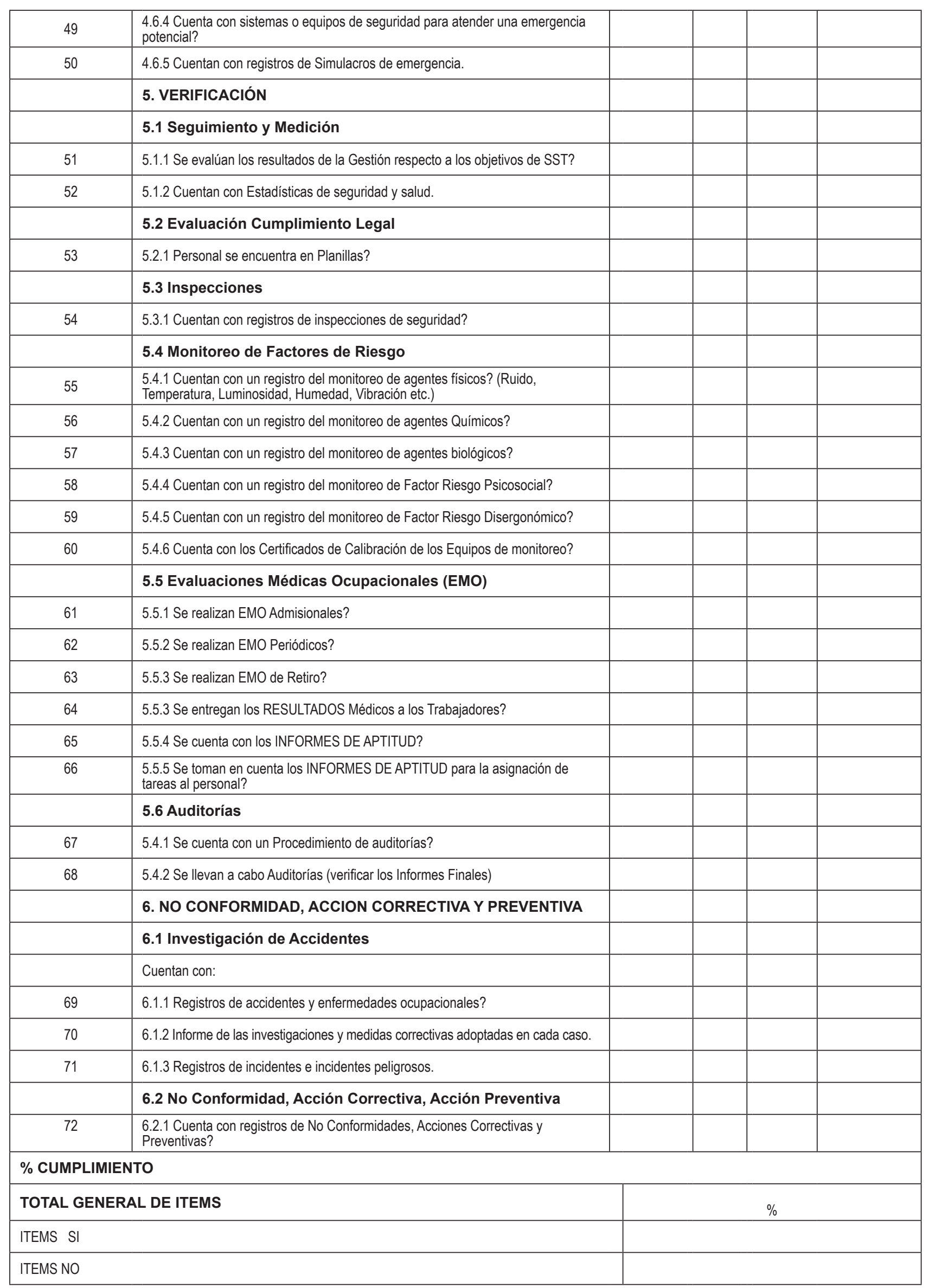

Fuente: Ley № 30222, publicado diario El Peruano julio 2014. (LEYDESEGURIDADSALUDTRABAJO-29783.pdf, s. f.) 
Apéndice 2. diagnostico D.S 0052012 TR - gestión de SST.

\begin{tabular}{|c|c|c|c|c|c|c|c|c|c|}
\hline & & & & & & Elaborado por: F.Falc & & & \\
\hline & DIAGNOS & STICO DE & E LINEA BASE D.S. 005 2012-TR & & & Revisado por: & & & \\
\hline & & & & & & Código: SSOMA & & & \\
\hline & & & & & & Fecha: & & & \\
\hline FECHADE INICIO: & & & & ORDEN DE IN & SPECCION & & & & \\
\hline RAZON SOCIAL: & & & & RUC: & & & & & \\
\hline DOMICILIO FISCA & & & & DISTRITO: & & & & & \\
\hline PROVINCIA: & & & & DEPARTAMEI & NTO: & & & & \\
\hline DISTRITO: & & & & & & & & & \\
\hline DEPARTAMENTO: & & & & & & & & & \\
\hline OTROS DATOS: & & & & & & & & & \\
\hline $\begin{array}{l}\text { DOC } / \\
\text { REGISTRO }\end{array}$ & DOC.LEGAL & $\begin{array}{c}\mathbf{N}^{\circ} \\
\text { Artículo }\end{array}$ & EXTRACTO DEL ARTICULO A CUMPLIR & EMPRESA & RIESGO & $\begin{array}{c}\text { PERIODICIDAD } \\
\text { MONITOREO }\end{array}$ & PLAZO & $\%$ & OBSERVACIONES \\
\hline $\begin{array}{l}\text { Manual del } \\
\text { Sistema de } \\
\text { Gestión }\end{array}$ & \begin{tabular}{|l|} 
Decreto \\
supremo. \\
005-2012 TR
\end{tabular} & $\begin{array}{l}\text { Artículo } \\
25^{\circ}\end{array}$ & $\begin{array}{l}\text { Cuenta con un sistema de SST en función a la } \\
\text { actividad o rubro que desarrolla. }\end{array}$ & & Todos & Anual & & & --- \\
\hline Política del SIG & \begin{tabular}{|l|} 
Decreto \\
supremo. \\
005-2012 TR
\end{tabular} & \begin{tabular}{l|} 
Artículo \\
$26^{\circ}$
\end{tabular} & $\begin{array}{l}\text { La organización avala que la gestión de SST haya } \\
\text { sido comunicada a todos sus trabajadores.. }\end{array}$ & & Todos & Anual & & & -- \\
\hline $\begin{array}{l}\text { Manual del } \\
\text { Sistema de } \\
\text { Gestión }\end{array}$ & \begin{tabular}{|l|} 
Decreto \\
supremo. \\
005-2012 TR \\
\end{tabular} & $\begin{array}{l}\text { Artículo } \\
26^{\circ}\end{array}$ & $\begin{array}{l}\text { Luego de la comunicación la organización deberá } \\
\text { definir qué área mantendrá el seguimiento de la } \\
\text { Gestión. }\end{array}$ & & Todos & Anual & & & --- \\
\hline-- & \begin{tabular}{|l|} 
Decreto \\
supremo. \\
005-2012 TR \\
\end{tabular} & \begin{tabular}{l|} 
Artículo \\
$26^{\circ}$
\end{tabular} & $\begin{array}{l}\text { Contar con supervisores de área en la organización } \\
\text { para el seguimiento de la gestión. }\end{array}$ & & Todos & Anual & & & -- \\
\hline $\begin{array}{l}\text { Comunicaciones } \\
\text { Internas }\end{array}$ & \begin{tabular}{|l|} 
Decreto \\
supremo. \\
005-2012 TR
\end{tabular} & $\begin{array}{l}\text { Artículo } \\
26^{\circ}\end{array}$ & $\begin{array}{l}\text { Promover la cooperación y la comunicación a todo } \\
\text { el personal, representantes de trabajadores y los } \\
\text { miembros de sindicato, con ello los elementos serán } \\
\text { factibles. }\end{array}$ & & Todos & Anual & & & -- \\
\hline--- & \begin{tabular}{|l|} 
Decreto \\
supremo. \\
005-2012 TR \\
\end{tabular} & \begin{tabular}{|l|} 
Artículo \\
$26^{\circ}$
\end{tabular} & $\begin{array}{l}\text { Cumplir los principios de los Sistemas de Gestión } \\
\text { de la SST dadas en el reglamento. }\end{array}$ & & Todos & Anual & & & -- \\
\hline Política del SIG & \begin{tabular}{|l|} 
Decreto \\
supremo. \\
005-2012 TR \\
\end{tabular} & $\begin{array}{l}\text { Artículo } \\
26^{\circ}\end{array}$ & $\begin{array}{l}\text { Contar y evaluar periódicamente la política, tener } \\
\text { un programa de SST con objetivos y metas que } \\
\text { puedan ser medidas y evidenciadas. }\end{array}$ & & Todos & Anual & & & -- \\
\hline Matriz IPER & \begin{tabular}{|l|} 
Decreto \\
supremo. \\
005-2012 TR \\
\end{tabular} & $\begin{array}{l}\text { Artículo } \\
26^{\circ}\end{array}$ & $\begin{array}{l}\text { Contar con IPERC de cada una de las tareas y } \\
\text { procesos. }\end{array}$ & & Todos & Anual & & & -- \\
\hline $\begin{array}{l}\text { Programa de } \\
\text { Sensibilización }\end{array}$ & \begin{tabular}{|l|} 
Decreto \\
supremo. \\
005-2012 TR \\
\end{tabular} & $\begin{array}{l}\text { Artículo } \\
26^{\circ}\end{array}$ & $\begin{array}{l}\text { Contar con Planes y programas de salud y el } \\
\text { sistema de monitoreo de su cumplimiento. }\end{array}$ & & Todos & Anual & & & -- \\
\hline $\begin{array}{l}\text { Comité de } \\
\text { Seguridad y Salud } \\
\text { en el Trabajo }\end{array}$ & \begin{tabular}{|l|} 
Decreto \\
supremo. \\
005-2012 TR
\end{tabular} & $\begin{array}{l}\text { Artículo } \\
26^{\circ}\end{array}$ & $\begin{array}{l}\text { Generar registros que demuestren la participación } \\
\text { de los trabajadores y los representantes en la } \\
\text { implementación del sistema, su Politica y en las } \\
\text { reuniones de Comités de SST. }\end{array}$ & & Todos & Anual & & & --- \\
\hline $\begin{array}{l}\text { Presupuesto } \\
\text { ASAM }\end{array}$ & \begin{tabular}{|l|} 
Decreto \\
supremo. \\
005-2012 TR \\
\end{tabular} & $\begin{array}{l}\text { Artículo } \\
26^{\circ}\end{array}$ & \begin{tabular}{|l|} 
Proporcionar los recursos o contar con registro de \\
las inversiones ejecutadas para cumplir los planes y \\
programas preventivos de SST.
\end{tabular} & & Todos & Anual & & & --- \\
\hline Servicio de SST & \begin{tabular}{|l|} 
Decreto \\
supremo. \\
005-2012 TR
\end{tabular} & $\begin{array}{l}\text { Artículo } \\
26^{\circ} \mathrm{A}\end{array}$ & $\begin{array}{l}\text { Si cuenta con una empresa especializada para la } \\
\text { gestión, implementación, monitoreo y cumplimiento } \\
\text { de seguridad y salud en el trabajo, no exime a los } \\
\text { empleadores de su responsabilidad. }\end{array}$ & & Todos & Anual & & & -- \\
\hline Servicio de SST & \begin{tabular}{|l|} 
Decreto \\
supremo. \\
005-2012 TR \\
\end{tabular} & $\begin{array}{l}\text { Artículo } \\
26^{\circ} \mathrm{A}\end{array}$ & $\begin{array}{l}\text { Para las funciones Comité de Seguridad y Salud en } \\
\text { el Trabajo, el empleador no podrá tercerizar. }\end{array}$ & & Todos & Anual & & & --- \\
\hline Servicio de SST & \begin{tabular}{|l|} 
Decreto \\
supremo. \\
005-2012 TR \\
\end{tabular} & $\begin{array}{l}\text { Artículo } \\
26^{\circ} \mathrm{A}\end{array}$ & $\begin{array}{l}\text { En caso de tercerización el empleador comunicará } \\
\text { de ello a sus trabajadores de manera oportuna } \\
\text { especificando que abarca estas funciones. } \\
\end{array}$ & & Todos & Anual & & & --- \\
\hline Servicio de SST & \begin{tabular}{|l|} 
Decreto \\
supremo. \\
005-2012 TR
\end{tabular} & $\begin{array}{l}\text { Artículo } \\
26^{\circ} \mathrm{A}\end{array}$ & $\begin{array}{l}\text { Si terceriza la empresa si puede facilitar el } \\
\text { cumplimiento de las funciones del Comité o } \\
\text { Supervisor de Seguridad y Salud en el Trabajo y, o } \\
\text { Subcomités. }\end{array}$ & & Todos & Anual & & & --- \\
\hline $\begin{array}{l}\text { Programa Anual } \\
\text { de Capacitaciones }\end{array}$ & \begin{tabular}{|l|} 
Decreto \\
supremo. \\
005-2012 TR
\end{tabular} & $\begin{array}{l}\text { Artículo } \\
27^{\circ}\end{array}$ & $\begin{array}{l}\text { El empleador de mostrará la capacitación y que } \\
\text { estos sean asumidos por el empleador. }\end{array}$ & & Todos & Anual & & & \\
\hline
\end{tabular}




\begin{tabular}{|c|c|c|c|c|c|c|}
\hline \begin{tabular}{l|} 
Programa Anual \\
de Capacitaciones
\end{tabular} & \begin{tabular}{|l|} 
Decreto \\
supremo. \\
005-2012 TR \\
\end{tabular} & $\begin{array}{l}\text { Artículo } \\
28^{\circ}\end{array}$ & $\begin{array}{l}\text { La capacitación bajo ningún caso el costo de la } \\
\text { capacitación recae sobre los trabajadores. }\end{array}$ & Todos & Anual & -- \\
\hline $\begin{array}{l}\text { Registros de } \\
\text { capacitaciones }\end{array}$ & \begin{tabular}{|l|} 
Decreto \\
supremo. \\
005-2012 TR \\
\end{tabular} & $\begin{array}{l}\text { Artículo } \\
29^{\circ}\end{array}$ & $\begin{array}{l}\text { Es responsabilidad del empleador capacitar a todos } \\
\text { los miembros de la organización. }\end{array}$ & Todos & Anual & -- \\
\hline $\begin{array}{l}\text { Recomendaciones } \\
\text { de SST }\end{array}$ & \begin{tabular}{|l|} 
Decreto \\
supremo. \\
005-2012 TR
\end{tabular} & $\begin{array}{l}\text { Artículo } \\
30^{\circ}\end{array}$ & $\begin{array}{l}\text { En el contrato de trabajo, deberá incluir las } \\
\text { recomendaciones de SST, sea física o digital, a más } \\
\text { tardar, el primer día de labores. }\end{array}$ & Todos & $\begin{array}{l}\text { Cuando se } \\
\text { requiera }\end{array}$ & -- \\
\hline Viáticos & \begin{tabular}{|l|} 
Decreto \\
supremo. \\
005-2012 TR
\end{tabular} & $\begin{array}{l}\text { Artículo } \\
31^{\circ}\end{array}$ & $\begin{array}{l}\text { El empleador otorgará facilidades económicas } \\
\text { y licencias con goce del inciso d) del Artículo } 35^{\circ} \\
\text { de la Ley 29783: costos del traslado y gastos de } \\
\text { alimentación y alojamiento, si es fuera del lugar } \\
\text { de trabajo o en una localidad o región distinta a } \\
\text { aquélla. }\end{array}$ & Todos & Anual & -- \\
\hline $\begin{array}{l}\text { Política del SIG } \\
\text { RISST } \\
\text { Matriz IPER } \\
\text { Mapa de Riesgo } \\
\text { PASST }\end{array}$ & \begin{tabular}{|l|} 
Decreto \\
supremo. \\
005-2012 TR
\end{tabular} & $\begin{array}{l}\text { Artículo } \\
32^{\circ}\end{array}$ & $\begin{array}{l}\text { El empleador tiene exhibido lo siguiente: } \\
\text { a.-La política y objetivos en SST } \\
\text { b.-RISST } \\
\text { c.-IPERC identificación de peligros, evaluación de } \\
\text { riesgos y sus medidas de control. } \\
\text { d.-El mapa de riesgo. } \\
\text { e.-La planificación de la actividad preventiva. } \\
\text { f.-El Programa Anual de SST }\end{array}$ & Todos & Anual & -- \\
\hline Formatos del SIG & \begin{tabular}{|l|} 
Decreto \\
supremo. \\
005-2012 TR
\end{tabular} & $\begin{array}{l}\text { Artículo } \\
33^{\circ}\end{array}$ & $\begin{array}{l}\text { a) Los registros de las investigaciones } \\
\text { de accidentes de trabajo, enfermedades } \\
\text { ocupacionales, incidentes peligrosos y otros } \\
\text { incidentes y las medidas correctivas. } \\
\text { b) Evidenciar el manejo, control y registro de } \\
\text { exámenes médicos ocupacionales. } \\
\text { c) Registro del monitoreo de agentes físicos, } \\
\text { químicos, biológicos, psicosociales disergonómico. } \\
\text { d) Registro de inspecciones del sistema. } \\
\text { e) Estadísticas SST } \\
\text { f) Evidencia de contar con elementos de emergencia } \\
\text { g) Registro de inducción, capacitación, } \\
\text { entrenamiento y simulacros. } \\
\text { h) Registro de auditorías internas y externas. }\end{array}$ & Todos & Anual & \\
\hline--- & \begin{tabular}{|l|} 
Decreto \\
supremo. \\
005-2012 TR
\end{tabular} & $\begin{array}{l}\text { Artículo } \\
34^{\circ}\end{array}$ & $\begin{array}{l}\text { Las micro, pequeñas y medianas empresas } \\
\text { (MIPYME) u otras que no tengan labores de alto } \\
\text { riesgo el MTPE indica simplificación se encuentran } \\
\text { en RM 085-2013-TR. }\end{array}$ & Todos & Anual & \\
\hline Formatos del SIG & \begin{tabular}{|l|} 
Decreto \\
supremo. \\
005-2012 TR
\end{tabular} & $\begin{array}{l}\text { Artículo } \\
35^{\circ}\end{array}$ & $\begin{array}{l}\text { Los registros en caso de enfermedades } \\
\text { ocupacionales serán conservados por } 20 \text { años; } \\
\text { los registros de accidentes de trabajo, incidentes } \\
\text { peligrosos serán conservados por } 10 \text { años; y los } \\
\text { demás registros por } 5 \text { años posteriores al suceso. } \\
\text { Pueden sr en físico o digital.. }\end{array}$ & Todos & Anual & --- \\
\hline $\begin{array}{l}\text { Comité de } \\
\text { Seguridad y Salud } \\
\text { en el Trabajo }\end{array}$ & \begin{tabular}{|l|} 
Decreto \\
supremo. \\
005-2012 TR \\
\end{tabular} & $\begin{array}{l}\text { Artículo } \\
38^{\circ}\end{array}$ & $\begin{array}{l}\text { El empleador mostrara como se encuentra } \\
\text { realizando las funciones el comité y tener el } \\
\text { reconocimiento de los representantes. }\end{array}$ & Todos & Anual & --- \\
\hline Comité de SST & \begin{tabular}{|l|} 
Decreto \\
supremo. \\
005-2012 TR \\
\end{tabular} & $\begin{array}{l}\text { Artículo } \\
40^{\circ}\end{array}$ & $\begin{array}{l}\text { Demostrar con registros, documentos u otros la } \\
\text { ejecución de las funciones del comité de SST. }\end{array}$ & Todos & Anual & --- \\
\hline Comité de SST & \begin{tabular}{|l|} 
Decreto \\
supremo. \\
005-2012 TR \\
\end{tabular} & $\begin{array}{l}\text { Artículo } \\
43^{\circ}\end{array}$ & $\begin{array}{l}\text { Registros de entrega de identificación a los } \\
\text { miembros del comité de SST. }\end{array}$ & Todos & Anual & --- \\
\hline Comité de SST & \begin{tabular}{|l|} 
Decreto \\
supremo. \\
005-2012 TR \\
\end{tabular} & $\begin{array}{l}\text { Artículo } \\
48^{\circ}\end{array}$ & $\begin{array}{l}\text { El empleador designara mediante votación a los } \\
\text { representantes, titulares y suplentes ante el Comité } \\
\text { de SST, entre el personal de dirección y confianza y } \\
\text { elegidos en la instalación. }\end{array}$ & Todos & Anual & --- \\
\hline Libro de Actas & \begin{tabular}{|l|} 
Decreto \\
supremo. \\
005-2012 TR
\end{tabular} & $\begin{array}{l}\text { Artículo } \\
49^{\circ}\end{array}$ & $\begin{array}{l}\text { Registros del proceso de elecciones de los } \\
\text { representantes de los trabajadores ante el comité } \\
\text { de SST. } \\
\text { Si tiene organización sindical mayoritaria, esta es } \\
\text { la responsable. Revisar libro de actas y libro de } \\
\text { seguridad }\end{array}$ & Todos & Anual & --- \\
\hline Política SIG & \begin{tabular}{|l|} 
Decreto \\
supremo. \\
005-2012 TR \\
\end{tabular} & $\begin{array}{l}\text { Artículo } \\
55^{\circ}\end{array}$ & $\begin{array}{l}\text { Los acuerdos de las reuniones del Comité de SST, } \\
\text { deberán ser ejecutados y demostrados ante la } \\
\text { autoridad. }\end{array}$ & Todos & Anual & --- \\
\hline Comité de SST & \begin{tabular}{|l|} 
Decreto \\
supremo. \\
005-2012 TR
\end{tabular} & $\begin{array}{l}\text { Artículo } \\
62^{\circ}\end{array}$ & $\begin{array}{l}\text { Verificar mediante registro que los representantes } \\
\text { de los trabajadores o del Supervisor de SST es de } \\
\text { un (1) año como mínimo o } \\
\text { (2) años como máximo. }\end{array}$ & Todos & Anual & --- \\
\hline Comité de SST & \begin{tabular}{|l|} 
Decreto \\
supremo. \\
005-2012 TR \\
\end{tabular} & $\begin{array}{l}\text { Artículo } \\
63^{\circ}\end{array}$ & $\begin{array}{l}\text { Registros de capacitación a todos los miembros del } \\
\text { comité de SST }\end{array}$ & Todos & Anual & --- \\
\hline
\end{tabular}




\begin{tabular}{|c|c|c|c|c|c|c|}
\hline Comité de SST & \begin{tabular}{|l|} 
Decreto \\
supremo. \\
005-2012 TR
\end{tabular} & $\begin{array}{l}\text { Artículo } \\
64^{\circ}\end{array}$ & $\begin{array}{l}\text { En caso de vacancia de los cargos son suplidos } \\
\text { por el representante alterno, hasta la conclusión } \\
\text { del mandato y deberán ser informados al comité de } \\
\text { SST, mostrar registro }\end{array}$ & Todos & Anual & -- \\
\hline Comité de SST & \begin{tabular}{|l|} 
Decreto \\
supremo. \\
005-2012 TR \\
\end{tabular} & $\begin{array}{l}\text { Artículo } \\
67^{\circ}\end{array}$ & $\begin{array}{l}\text { Las reuniones del Comité de SST se deben } \\
\text { ejecutar en los horarios de trabajo.. }\end{array}$ & Todos & Anual & --- \\
\hline Comité de SST & \begin{tabular}{|l|} 
Decreto \\
supremo. \\
005-2012 TR
\end{tabular} & $\begin{array}{l}\text { Artículo } \\
68^{\circ}\end{array}$ & $\begin{array}{l}\text { Registros de las reuniones del Comité de SST } \\
\text { ordinaria } 1 \text { vez por mes, en día previamente fijado. } \\
\text { Extraordinaria, donde su Presidente, a solicitud de } \\
\text { al menos } 2 \text { de sus miembros, o en caso de ocurrir } \\
\text { un accidente mortal. }\end{array}$ & Todos & Anual & --- \\
\hline $\begin{array}{l}\text { Acta de Comité } \\
\text { SST }\end{array}$ & \begin{tabular}{|l|} 
Decreto \\
supremo. \\
005-2012 TR
\end{tabular} & $\begin{array}{l}\text { Artículo } \\
69^{\circ}\end{array}$ & \begin{tabular}{l|} 
Revisar el cumplimiento del quórum mínimo para \\
sesionar del Comité de SST mitad más 01 de los \\
integrantes. Caso contrario, verificar que se cumpla \\
los 8 días.
\end{tabular} & Todos & Anual & --- \\
\hline Comité de SST & \begin{tabular}{|l|} 
Decreto \\
supremo. \\
005-2012 TR \\
\end{tabular} & $\begin{array}{l}\text { Artículo } \\
72^{\circ}\end{array}$ & $\begin{array}{l}\text { Mostrar el informe anual del Comité de SST de sus } \\
\text { labores realizadas. }\end{array}$ & Todos & Anual & --- \\
\hline Política SIG & \begin{tabular}{|l|} 
Decreto \\
supremo. \\
005-2012 TR \\
\end{tabular} & $\begin{array}{l}\text { Artículo } \\
73^{\circ}\end{array}$ & \begin{tabular}{|l|} 
Demostrar que los miembros del Comité de SST \\
(representante de los trabajadores) hagan uso de \\
su licencia con goce de (30) dias naturales por año. \\
\end{tabular} & Todos & Anual & --- \\
\hline RISST & \begin{tabular}{|l|} 
Decreto \\
supremo. \\
005-2012 TR
\end{tabular} & $\begin{array}{l}\text { Artículo } \\
74^{\circ}\end{array}$ & $\begin{array}{l}\text { De tener más de } 20 \text { trabajadores mostrar el } \\
\text { Reglamento Interno de SST. }\end{array}$ & Todos & Anual & --- \\
\hline RISST & \begin{tabular}{|l|} 
Decreto \\
supremo. \\
005-2012 TR \\
\end{tabular} & $\begin{array}{l}\text { Artículo } \\
75^{\circ}\end{array}$ & $\begin{array}{l}\text { Cargo de entrega del Reglamento Interno de } \\
\text { SST y sus posteriores modificatorias a todos los } \\
\text { trabajadores. }\end{array}$ & Todos & Anual & -- \\
\hline $\begin{array}{l}\text { Evaluación Línea } \\
\text { Base }\end{array}$ & \begin{tabular}{|l|} 
Decreto \\
supremo. \\
005-2012 TR
\end{tabular} & $\begin{array}{l}\text { Artículo } \\
77^{\circ}\end{array}$ & $\begin{array}{l}\text { Registro o documentos que demuestren la } \\
\text { evaluación inicial de riesgos de cada puesto } \\
\text { de trabajo por empresa o personal competente } \\
\text { previamente consultas a los trabajadores y } \\
\text { miembros del Comité de SST. } \\
\end{array}$ & Todos & Anual & --- \\
\hline Matriz IPER & \begin{tabular}{|l|} 
Decreto \\
supremo. \\
005-2012 TR
\end{tabular} & $\begin{array}{l}\text { Artículo } \\
82^{\circ}\end{array}$ & $\begin{array}{l}\text { Revisan periódica de su IPERc (revisar registro). } \\
\text { Consultada con los trabajadores, organización } \\
\text { sindical o el Comité según el caso. }\end{array}$ & Todos & Anual & -- \\
\hline $\begin{array}{l}\text { Procedimiento } \\
\text { del SIG }\end{array}$ & \begin{tabular}{|l|} 
Decreto \\
supremo. \\
005-2012 TR \\
\end{tabular} & $\begin{array}{l}\text { Artículo } \\
83^{\circ}\end{array}$ & $\begin{array}{l}\text { Plan de preparación y respuesta ante situaciones de } \\
\text { emergencia y accidentes de trabajo. }\end{array}$ & Todos & Anual & --- \\
\hline $\begin{array}{l}\text { Procedimiento de } \\
\text { logística }\end{array}$ & \begin{tabular}{|l|} 
Decreto \\
supremo. \\
005-2012 TR \\
\end{tabular} & $\begin{array}{l}\text { Artículo } \\
84^{\circ}\end{array}$ & $\begin{array}{l}\text { Revisar los procedimientos y estándares de trabajo } \\
\text { acorde al rubro. }\end{array}$ & Todos & Anual & -- \\
\hline $\begin{array}{l}\text { Manual del } \\
\text { Sistema de } \\
\text { Gestión }\end{array}$ & \begin{tabular}{|l|} 
Decreto \\
supremo. \\
005-2012 TR
\end{tabular} & $\begin{array}{l}\text { Artículo } \\
86^{\circ}\end{array}$ & \begin{tabular}{|l|} 
Demostrar, cualitativa y cuantitativamente, la \\
ejecución de las necesidades de la gestión de SST \\
en de la organización.
\end{tabular} & Todos & Anual & -- \\
\hline \begin{tabular}{l|} 
Comité de \\
Seguridad y Salud \\
en el Trabajo
\end{tabular} & \begin{tabular}{|l|} 
Decreto \\
supremo. \\
005-2012 TR \\
\end{tabular} & $\begin{array}{l}\text { Artículo } \\
88^{*}\end{array}$ & $\begin{array}{l}\text { Las investigaciones deben ser realizadas por } \\
\text { el empleador, el Comité y/o Supervisor de SST, } \\
\text { verificar registros. }\end{array}$ & Todos & Anual & --- \\
\hline $\begin{array}{l}\text { Revisión por la } \\
\text { Dirección }\end{array}$ & \begin{tabular}{|l|} 
Decreto \\
supremo. \\
005-2012 TR \\
\end{tabular} & $\begin{array}{l}\text { Artículo } \\
90^{*}\end{array}$ & $\begin{array}{l}\text { Mostrar la revisión del sistema de gestión de la } \\
\text { seguridad y salud en el trabajo mínimo (1) vez al } \\
\text { año }\end{array}$ & Todos & Anual & -- \\
\hline $\begin{array}{l}\text { Revisión por la } \\
\text { Dirección }\end{array}$ & \begin{tabular}{|l|} 
Decreto \\
supremo. \\
005-2012 TR \\
\end{tabular} & $\begin{array}{l}\text { Artículo } \\
91^{*}\end{array}$ & $\begin{array}{l}\text { Comunicación de los resultados de los examinen de } \\
\text { ingreso y periódico a sus trabajadores. }\end{array}$ & Todos & Anual & --- \\
\hline $\begin{array}{l}\text { Servicio de } \\
\text { Medicina } \\
\text { Ocupacional }\end{array}$ & \begin{tabular}{|l|} 
Decreto \\
supremo. \\
005-2012 TR
\end{tabular} & $\begin{array}{l}\text { Artículo } \\
92^{*}\end{array}$ & $\begin{array}{l}\text { La concesión de puestos de trabajo, conforme a lo } \\
\text { establecido en el artículo } 51^{\circ} \text { de la Ley, demostrar } \\
\text { ejecución. }\end{array}$ & Todos & Anual & --- \\
\hline EPP & \begin{tabular}{|l|} 
Decreto \\
supremo. \\
005-2012 TR \\
\end{tabular} & $\begin{array}{l}\text { Artículo } \\
97^{*}\end{array}$ & $\begin{array}{l}\text { EPP que demuestren las medidas antropométricas } \\
\text { del trabajador que los utilizará. }\end{array}$ & Todos & Anual & -- \\
\hline $\begin{array}{l}\text { Programa Anual } \\
\text { de Capacitaciones }\end{array}$ & \begin{tabular}{|l|} 
Decreto \\
supremo. \\
005-2012 TR \\
\end{tabular} & $\begin{array}{l}\text { Artículo } \\
98^{*}\end{array}$ & $\begin{array}{l}\text { Demostrar que el comité de SST cuenta con área } \\
\text { para celebrar sus reuniones }\end{array}$ & Todos & Anual & -- \\
\hline $\begin{array}{l}\text { Exámenes médico } \\
\text { ocupacional }\end{array}$ & \begin{tabular}{|l|} 
Decreto \\
supremo. \\
005-2012 TR \\
\end{tabular} & $\begin{array}{l}\text { Artículo } \\
101^{*}- \\
102^{*} \\
\end{array}$ & \begin{tabular}{|l|} 
Verificar que se lleven a cabo los exámenes \\
médicos de ingreso, periódicos y de retiro o cambios \\
de puestos El costo será asumido por el empleador.
\end{tabular} & Todos & Bianual/Anual & --- \\
\hline Comité de SST & \begin{tabular}{|l|} 
Decreto \\
supremo. \\
005-2012 TR
\end{tabular} & $\begin{array}{l}\text { Artículo } \\
104^{\circ}\end{array}$ & $\begin{array}{l}\text { En el caso que existan cambios en las operaciones } \\
\text { y procesos, (artículo } 70^{\circ} \text { de la Ley), mostrar los } \\
\text { registros de la comunicación a los trabajadores o } \\
\text { actas del Comité o Supervisor, SST. }\end{array}$ & Todos & Anual & --- \\
\hline \begin{tabular}{l|} 
Reglamento \\
Interno de Trabajo
\end{tabular} & \begin{tabular}{|l|} 
Decreto \\
supremo. \\
005-2012 TR \\
\end{tabular} & $\begin{array}{l}\text { Artículo } \\
109^{\circ}\end{array}$ & $\begin{array}{l}\text { Verificar que ell Reglamento Interno de Trabajo } \\
\text { del empleador indique las sanciones en caso de } \\
\text { incumplimientos a la SST (ART 79º de la Ley. }\end{array}$ & Todos & Anual & --- \\
\hline Comité de SST & \begin{tabular}{|l|} 
Decreto \\
supremo. \\
005-2012 TR
\end{tabular} & $\begin{array}{l}\text { Artículo } \\
110^{\circ}\end{array}$ & $\begin{array}{l}\text { Las comunicaciones como mínimo de } 24 \text { horas en } \\
\text { caso de accidentes mortales, Incidentes Peligrosos } \\
\text { de ocurrido lo antes mencionado. }\end{array}$ & Todos & $\begin{array}{c}\text { Cuando sucede } \\
\text { un accidente } \\
\text { o enfermedad } \\
\text { ocupacional } \\
\end{array}$ & -- \\
\hline
\end{tabular}


REV. DEL INSTITUTO dE INVESTIGACIÓN FIGMMG-UNMSM 23(45) 2020

\begin{tabular}{|l|l|l|l|l|l|l|l|l|}
\hline-- & $\begin{array}{l}\text { Decreto } \\
\text { supremo. } \\
005-2012 \text { TR }\end{array}$ & $\begin{array}{l}\text { Artículo } \\
112^{\circ}\end{array}$ & Registrar en el T registro de tener un Comité de SST & Todos & $\begin{array}{l}\text { Cuando sucede } \\
\text { un accidente } \\
\text { o enfermedad } \\
\text { ocupacional }\end{array}$ & -- & \\
\hline
\end{tabular}

\begin{tabular}{|l|l|}
\hline TOTAL GENERAL DE ITEMS & $\%$ \\
\hline ITEMS SI & \\
\hline ITEMS NO & \\
\hline
\end{tabular}

Fuente. (DECRETO SUPREMO Nº05-2012-TR - Norma Legal Diario Oficial El Peruano, s. f.) 